\title{
SEDIMENTATION PATTERN OF SOILS SOUTH EL-AMIRIA, ALEXANDRIA GOVERNORATE, EGYPT
}

\author{
A. M. A. Zayed, H. M. A. El-Tapey and A. A. Al-Toukhy \\ Soils, Water and Environmental Research Institute, Agricultural Research Center, \\ Giza, Egypt
}

Received: Apr. 26,2021

Accepted: Jun. 29, 2021

\begin{abstract}
The current study aims to identify the sedimentation pattern of soils south El-Amiria, Alexandria Governorate, Egypt. This includes study of grain size parameters, depositional environments and hydrodynamic conditions of these soils. The study area is located between longitudes $29^{\circ} 47^{\prime} 55^{\prime \prime}$ and $30^{\circ} 30^{\prime} 05^{\prime \prime}$ East and latitudes $29^{\circ} 29^{\prime} 30^{\prime \prime}$ and $30^{\circ} 30^{\prime} 05^{\prime \prime}$ North and comprise an area of about 571168 Feddan. The area is characterized by two main physiographic units namely Lacustrine plains and Windblown sand. The obtained results indicated that:
\end{abstract}

I. Soils of lacustrine plains appeared that:

1-Grain size parameters as follows:

- The fine silt was the predominant constituent of median (Md) as average size.

- Graphic mean (Mz), generally, differed from fine to medium and coarse silt.

- Measure of uniformity appeared very poorly sorted values $\left(\sigma_{1}\right)$ which indicated that the sediments are transported and deposited by water.

- Measure of symmetry cleared that, the sediments have values of strongly coarse skewed (SKI). These values indicate that, the examined sediments have a tail of coarse grains.

- Measure of peakedness showed that, the distribution of grains between platy \& very platykurtic and lepto \& very leptokurtic.

2- The depositional environments of lacustrine plain soils were fluvial or deltaic.

3- The hydrodynamic conditions, in general, were pelagic suspension ( $T$ ).

II. Soils of windblown sand showed that:

1-Grain size parameters as follows:

- Coarse sand was a median (Md) and graphic mean (Mz).

- Moderately and moderately well sorted values $\left(\sigma_{1}\right)$ represented the measure of uniformity. This indicated that, sediments are transported and deposited under a combined action of both water and wind.

- Strongly fine skewed class was the measure of a symmetry, which indicated that the examined sediments have a tail of fine grains.

- Lepto and very leptokurtic were the measure of peakedness.

2-The depositional environment of soil of windblown sand was either fluvial or deltaic.

3-The mechanism of transportation was rolling $(\mathrm{N}-\mathrm{O})$.

Key words: Statistical size parameter, sorting, skewness, kurtosis.

\section{INTRODUCTION}

Sandy and sandy calcareous soils in Egypt are a large area for agricultural horizontal expansion. Studying of physical and sedimentation pattern are considered as fundamental bases for a successful reclamation and high utilization.

According to Zayed et al (2020) the studied area has two main physiographic units, namely lacustrine plains and windblown sand (Map, 1). 


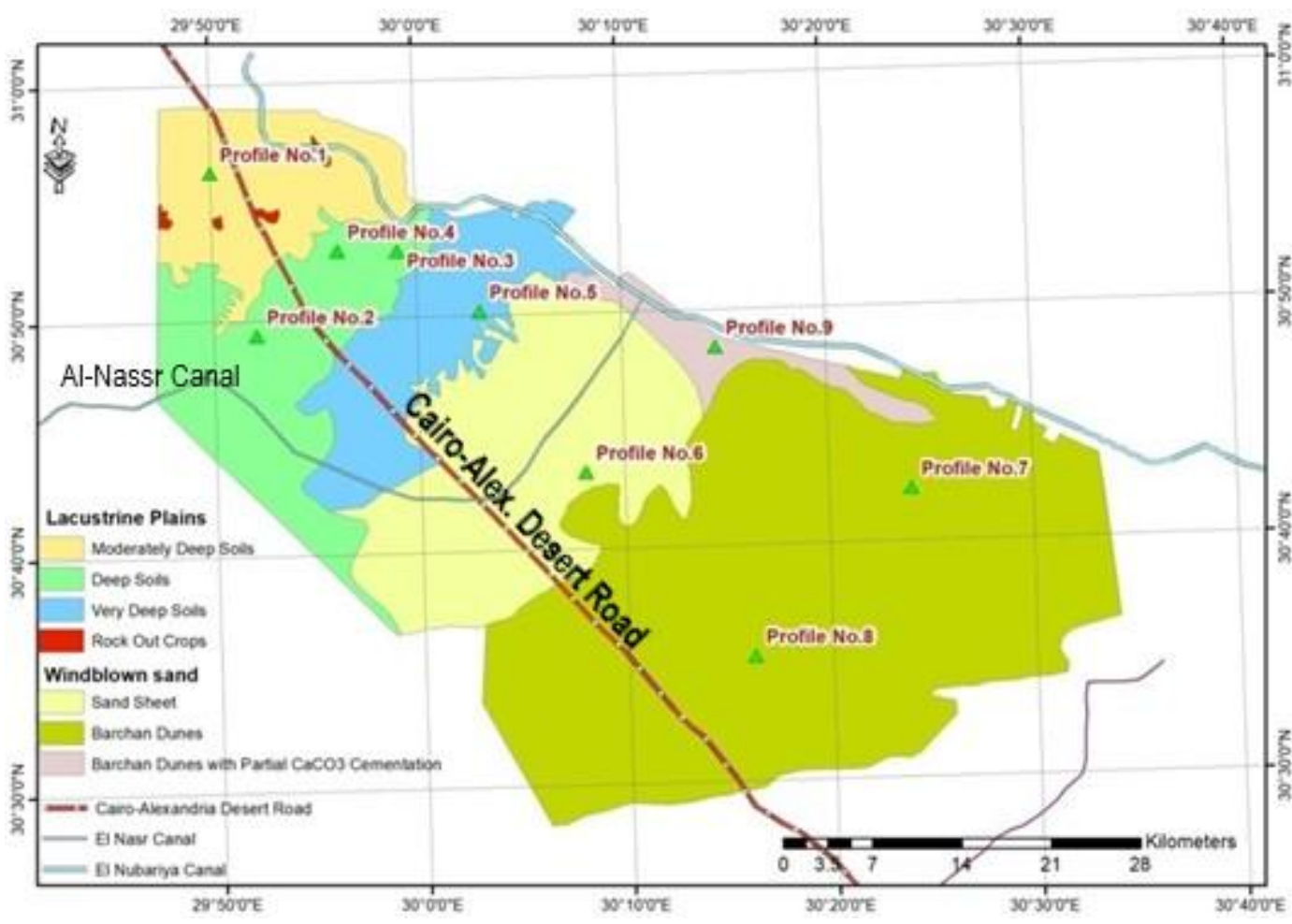

Map (1): Location of the studied area.

Several investigations have dealt with the interpretation of depositional environments from the particle size distribution such as Doeglas (1946), Passega (1957) and Folk and Ward (1957). Folk (1980) stated that, the most commonly used method involves plotting the cumulative curve of the sample and reading the diameter represented by various cumulative percentages. In this method, much more accurate results are obtained if one lots the cumulative curve on probability paper because of the superior accuracy of extrapolation and interpolation.

The common procedures in sedimentological studies are the different grain size parameters of Folk and Ward (1957) which include graphic mean size $(\mathrm{Mz})$, sorting ( $\sigma 1)$, skewness (Skl) and kurtosis (KG). Discrimination between different mechanisms and environments of deposition are suggested by Sahu (1964). On the other hand, the hydrodynamic conditions of sedimentation are suggested by C-M pattern of Passega (1957 and 1964).

The current work aims to study the sedimentation pattern for the soils of South El-Amiria, Egypt, using their particle size distribution and cumulative curves.

\section{MATERIALS AND METHODS}

Nine soil profiles were chosen to represent the two physiographic units of the studied area South El-Amiria, Alexandria governorate. Twenty-two soil samples were collected from these profiles, air-dried, crushed and sieved through 2-mm sieve. Mechanical analysis was carried out according to Burt (2004). Grain size distribution of sand fraction was carried out using fine sets of sieve diameter according to Piper (1950).

Sahu (1964) and Visher (1969) stated that, examination of the cumulative 
curves of non-clay fraction is helpful because the setting properties of claysized particles (< $0.002 \mathrm{~mm})$ are uncertain. So, statistical evaluation of the mechanical analysis data particularly with regard to dominant non-clay fraction (sand and silt) was performed.

Cumulative percentages were plotted against phi-diameter on arithmetic probability paper and eight diameters were estimated against plotted percentiles: ф11, ф55, ф16, ф25, ф50, ф75, op84 and p95\%, according to Griffiths (1967).

The statistical grain size parameter was then determined according to the formulae of Folk and Ward (1957), as follows:

1- Graphic mean: $\mathrm{M}_{\mathbf{z}}=(\phi 16+\phi \mathbf{5 0}+$ op 84) $/ 3$

2- Inclusive Graphic Standard Deviation: $\sigma_{1}=\frac{\phi 84-\phi 16}{4}+\frac{\phi 95-\phi 5}{6.6}$

3- Inclusive Graphic Skewness: $\mathbf{S k}_{\mathbf{l}}=$ $\frac{\phi 16+\phi 84-2 \phi 50}{2(\phi 84-\phi 16)}+\frac{\phi 5+\phi 95-2 \phi 50}{2(\phi 95-\phi 5)}$

4- The Graphic Kurtosis: $K_{G}=$ $\frac{\phi 95-\phi 5}{2.44(\phi 75-\phi 25)}$

Median: $M d$ is the diameter corresponding to the $50 \%$ mark on the cumulative curves.

The depositional mechanisms and their environments were studied according to Sahu (1964), through the following equations:

$\mathrm{Y}$ aeol : beoch $=-3.5688 \mathrm{M}_{\mathrm{z}}+3.7016 \sigma_{1}{ }^{2}$ - $2.0766 \mathrm{Sk}_{\mathrm{l}}+3.1135 \mathrm{~K}_{\mathrm{G}}$

$Y$ beach : sh.mar. $=15.6534 \mathrm{M}_{\mathrm{z}}+65.709$ $\sigma_{1}{ }^{2}+18.1071 \mathrm{Sk}_{1}+18.5043 \mathrm{~K}_{\mathrm{G}}$

Y sh : mar.: fluv. $=0.2852 \mathrm{M}_{\mathrm{z}}-8.7604 \sigma_{1}{ }^{2}$ $-4.8932 \mathrm{Sk}_{1}+0.0482 \mathrm{~K}_{\mathrm{G}}$

$Y$ fluv. : turb. $=0.7215 \mathrm{M}_{\mathrm{z}}-0.4030 \sigma_{1}{ }^{2}+$ $6.7322 \mathrm{Sk}_{\mathrm{I}}+5.2927 \mathrm{~K}_{\mathrm{G}}$

The C-M pattern of the examined soil sediments was used as a tool for indicating the hydrodynamic conditions of sedimentation as suggested by Passega (1957 and 1964).

\section{RESULTS AND DISCUSSION}

The sedimentological properties of the soils in studied area are investigated through their particle size distribution and cumulative curves presented in Table (1) and illustrated in Figs (1 and 2). The different parameters included grain size parameters, depositional environments and hydrodynamic conditions for each physiographic unit could be discussed as follows:

1-Grain Size parameters: According to Folk and Ward (1957) and Folk (1980), there are four parameters of grain size are suggested as follows:

1.1. Measures of average size:

a) Median (Md): Half of the particles by weight are coarser than the median and half are finer. It is the diameter corresponding to $50 \%$ mark on the cumulative curve and is expressed in dp $\left(\mathrm{Md}_{\phi}\right)$ according to Folk (1980) which presented in Table (2).

Soils of Lacustrine plains record values of median as follows: fine silt in profiles 1,2,3 and surface layer of profile 4 , medium silt in two subsurface layer of profile 4, coarse sand in upper two layers of profile 5 while the deepest layer appear medium value of very fine sand.

Soils of windblown sand (profiles 6,7 , 8 and 9) showed coarse sand as median

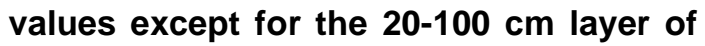
profile 7 which is very coarse sand.

b) Graphic mean (Mz): It is much superior to the median because it based on three points $[\mathrm{Mz}=(\phi p 16+\phi$ 50+ $\phi$ 84) 13] and give a better overall picture (Folk, 1980). Data of graphic mean (Mz) are recorded in Table (3).

Soils of lacustrine plains showed graphic mean values as follows: fine silt in soils of profile 3 and subsurface layer 
A. M. A. Zayed, et al.,

of profile 2, coarse silt in soil of profile 4 and deepest layer of profile 1 , medium silt in upper two layers of profile 1 and upper layer of profile 2. Graphic mean decreases with depth in soils of profile 5 from medium sand to very fine sand.

Soils of windblown sand have coarse sand in all representative profiles and their layers as a graphic mean (Mz).

\subsection{Measure of uniformity:}

According to Folk (1980), several measures are available for measuring uniformity or sorting. The Inclusive Graphic Standard Deviation ( $\sigma 1)$ includes $90 \%$ of the distribution and is the best overall measure of sorting. Data of sorting $\phi$ recorded in Table (3).

Table (1): Particle size distribution of the representative soil profiles.

\begin{tabular}{|c|c|c|c|c|c|c|c|c|c|}
\hline \multirow{3}{*}{ Landform } & \multirow{3}{*}{$\begin{array}{c}\text { Profile } \\
\text { No. }\end{array}$} & \multirow{3}{*}{$\begin{array}{l}\text { Depth } \\
\text { Cm. }\end{array}$} & \multicolumn{7}{|c|}{ Particle size distribution (\%) } \\
\hline & & & \multicolumn{5}{|c|}{ Sand fractions ${ }^{*}(\mathrm{~mm})$} & \multirow{2}{*}{$\begin{array}{c}\text { Silt } \\
0.05-0.002 \\
(\mathrm{~mm})\end{array}$} & \multirow{2}{*}{$\begin{array}{l}\text { Clay } \\
<0.002 \\
(\mathrm{~mm})\end{array}$} \\
\hline & & & $\begin{array}{c}\text { V.c.S } \\
2-1\end{array}$ & $\begin{array}{c}\text { C.S } \\
1-0.5\end{array}$ & \begin{tabular}{|c|} 
M.S \\
$0.5-0.25$
\end{tabular} & $\begin{array}{c}\text { F.S } \\
0.25-0.10\end{array}$ & $\begin{array}{c}\text { V.F.S } \\
0.20-0.05\end{array}$ & & \\
\hline \multicolumn{10}{|c|}{ Lacustrine plains } \\
\hline \multirow{3}{*}{ Moderately deep } & \multirow{3}{*}{1} & $0-20$ & 2.65 & 6.15 & 9.71 & 4.35 & 7.05 & 52.97 & 17.12 \\
\hline & & $20-60$ & 2.65 & 7.36 & 9.40 & 3.00 & 3.60 & 55.12 & 18.87 \\
\hline & & $60-90$ & 3.85 & 10.15 & 10.00 & 2.50 & 1.52 & 52.90 & 19.08 \\
\hline \multirow{7}{*}{ Deep } & \multirow{2}{*}{2} & $0-50$ & 1.63 & 4.48 & 5.09 & 3.05 & 4.58 & 50.51 & 30.66 \\
\hline & & $50-110$ & 1.52 & 5.07 & 4.54 & 4.05 & 2.53 & 49.42 & 32.87 \\
\hline & \multirow{2}{*}{3} & $0-45$ & 1.80 & 3.95 & 4.23 & 4.02 & 4.05 & 48.18 & 33.77 \\
\hline & & $45-110$ & 2.00 & 5.00 & 4.50 & 2.50 & 1.02 & 50.21 & 34.77 \\
\hline & \multirow{3}{*}{4} & $0-30$ & 6.13 & 13.68 & 7.31 & 0.69 & 0.60 & 40.88 & 30.71 \\
\hline & & $30-70$ & 9.22 & 15.29 & 6.36 & 1.42 & 0.83 & 38.87 & 28.01 \\
\hline & & $70-130$ & 12.95 & 11.98 & 10.09 & 0.86 & 0.85 & 33.62 & 29.65 \\
\hline \multirow{3}{*}{ Very deep } & \multirow{3}{*}{5} & $0-30$ & 37.85 & 22.71 & 7.96 & 2.15 & 1.20 & 13.22 & 14.91 \\
\hline & & $30-80$ & 26.30 & 27.20 & 10.58 & 4.17 & 2.45 & 15.31 & 13.99 \\
\hline & & $80-160$ & 4.23 & 19.27 & 16.25 & 12.58 & 15.41 & 19.14 & 13.12 \\
\hline \multicolumn{10}{|c|}{ Windblown sand } \\
\hline \multirow{2}{*}{ Sand Sheet } & \multirow{2}{*}{6} & $0-50$ & 30.50 & 37.71 & 13.09 & 4.93 & 2.99 & 7.12 & 3.66 \\
\hline & & $50-100$ & 34.25 & 35.95 & 12.35 & 4.55 & 2.03 & 6.13 & 4.74 \\
\hline \multirow{4}{*}{ Barchan dunes } & \multirow{2}{*}{7} & $0-20$ & 39.55 & 34.32 & 12.79 & 5.23 & 4.78 & 2.27 & 1.06 \\
\hline & & $20-100$ & 58.06 & 27.16 & 6.78 & 2.23 & 2.60 & 2.11 & 1.06 \\
\hline & \multirow{2}{*}{8} & $0-20$ & 41.86 & 41.32 & 10.44 & 2.56 & 1.01 & 1.67 & 1.14 \\
\hline & & $20-100$ & 36.11 & 42.22 & 12.25 & 4.62 & 1.94 & 1.72 & 1.14 \\
\hline \multirow{3}{*}{$\begin{array}{c}\text { Barchan dunes } \\
\text { partial cemented } \\
\text { with } \mathrm{CaCO}_{3}\end{array}$} & \multirow{3}{*}{9} & $0-40$ & 24.77 & 37.46 & 14.31 & 6.12 & 0.70 & 8.72 & 7.92 \\
\hline & & $40-80$ & 28.22 & 37.42 & 15.03 & 5.24 & 0.10 & 10.12 & 3.87 \\
\hline & & $80-120$ & 37.54 & 33.97 & 12.29 & 2.33 & 1.45 & 6.61 & 5.81 \\
\hline
\end{tabular}

${ }^{\star}$ V.C.S: Very coarse sand, C.S: Coarse sand, M.S: Medium sand, F.S: Fine Sand, V.F.S: Very fine sand 


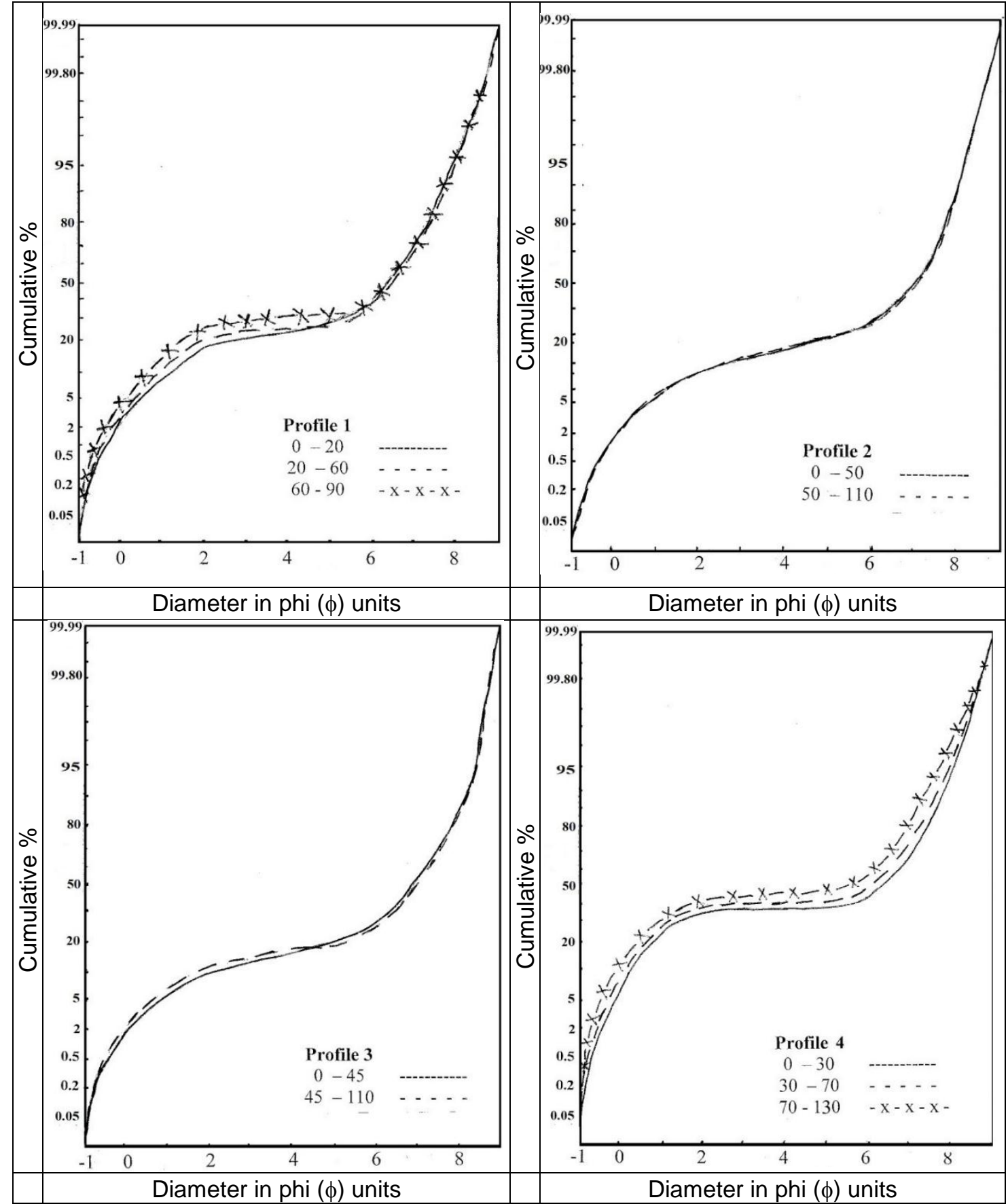

Fig (1): Cumulative frequency curves of sand and silt fractions of the studied Lacustrine plains soils. 
A. M. A. Zayed, et al.,

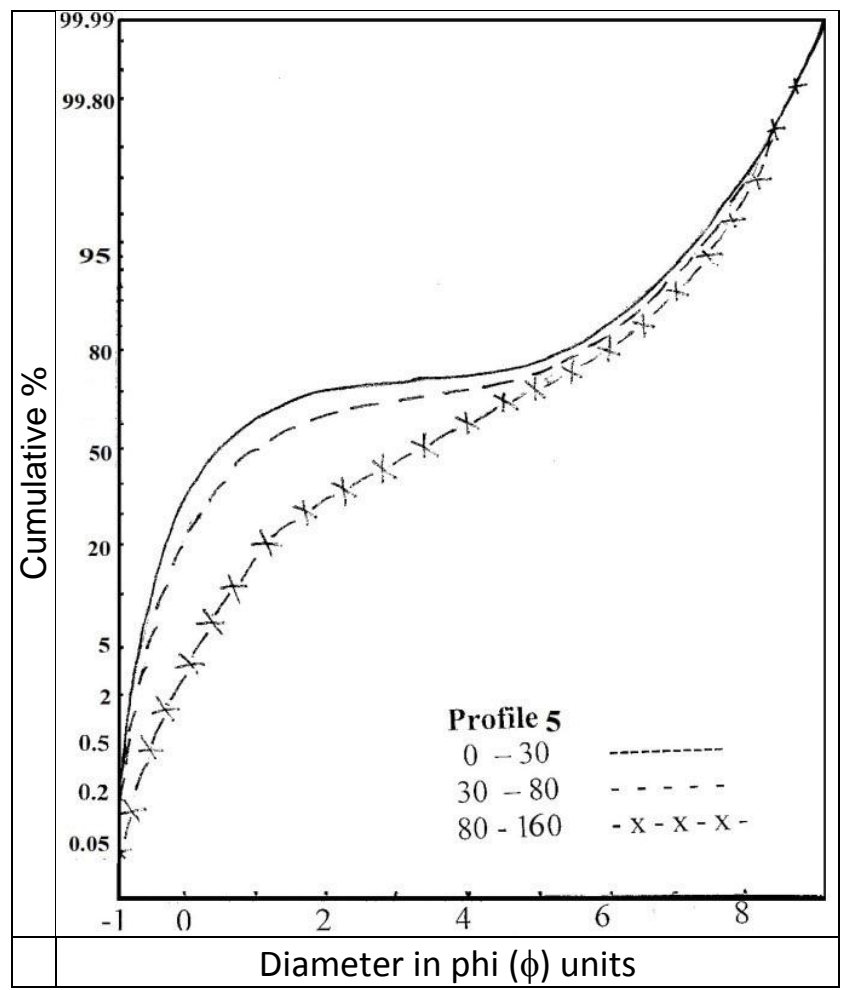

Fig (1): Cont.

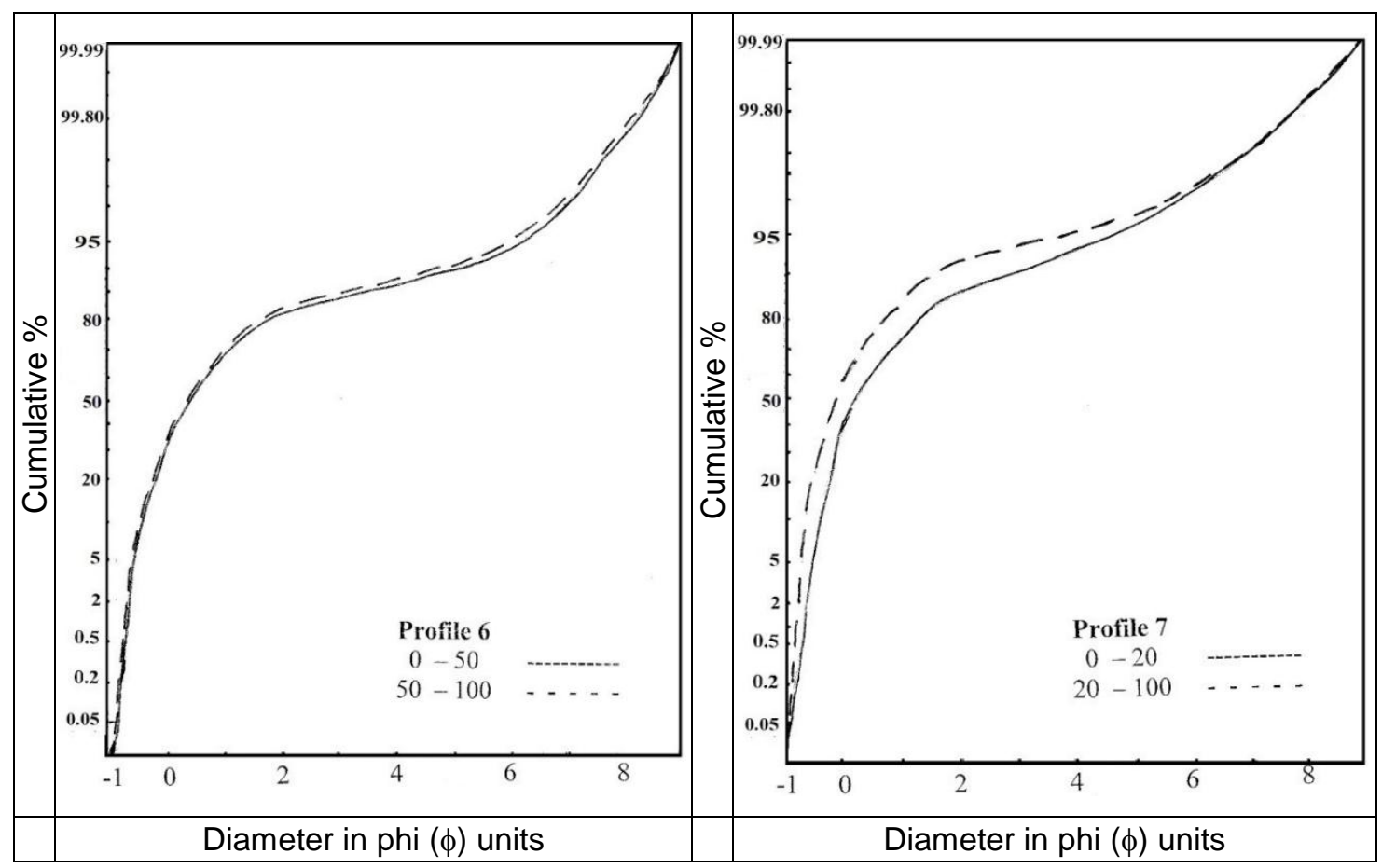

Fig (2): Cumulative frequency curves of sand and silt fractions of the studied Windblown soils. 


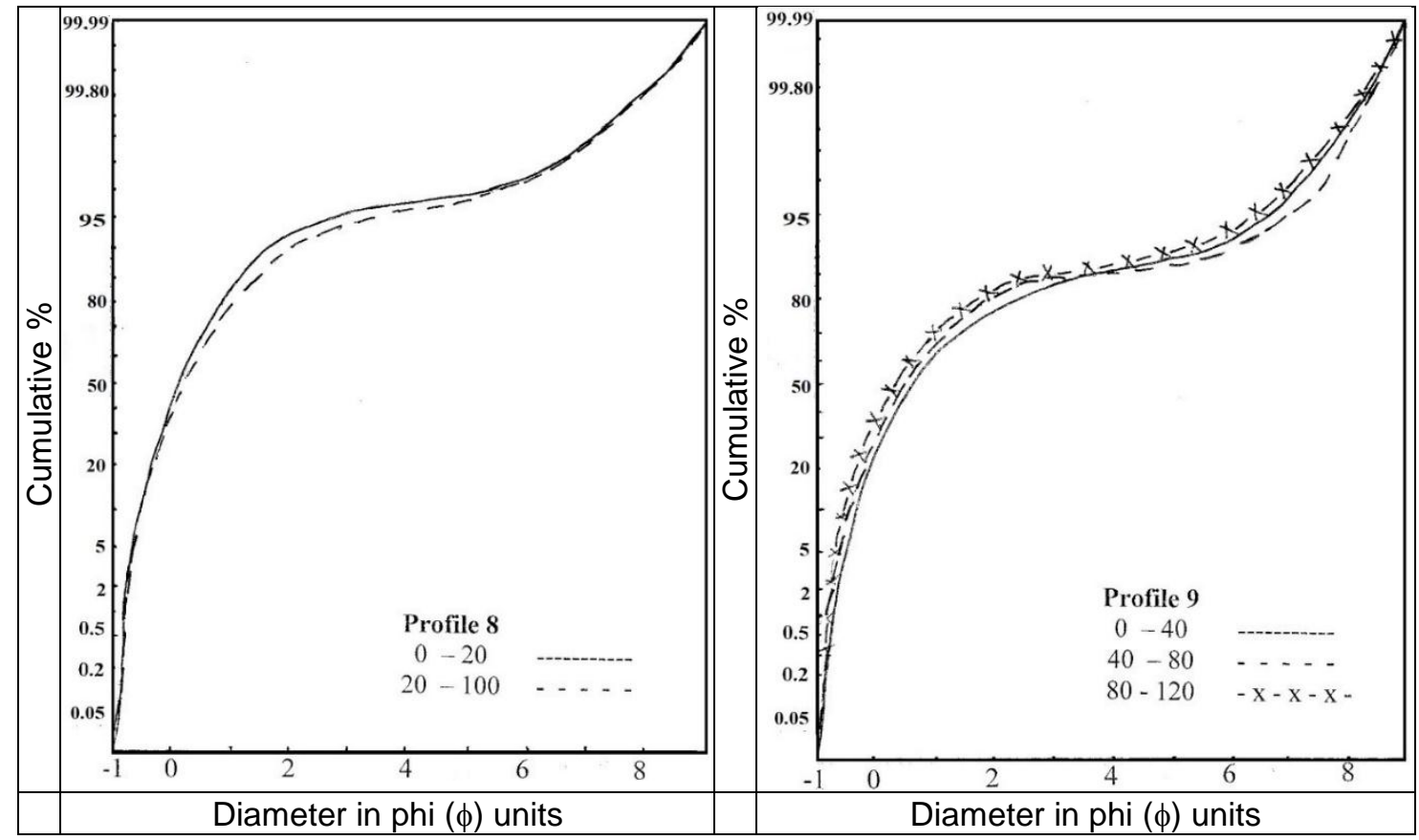

Fig (2): Cont.

Table (2): Phi-diameters $(\phi)$ against eight percentiles on arithmetic probability paper.

\begin{tabular}{|c|c|c|c|c|c|c|c|c|c|c|}
\hline \multirow{2}{*}{ Landform } & \multirow{2}{*}{$\begin{array}{c}\text { Profile } \\
\text { No. }\end{array}$} & \multirow{2}{*}{$\begin{array}{c}\text { Depth } \\
\text { Cm. }\end{array}$} & \multicolumn{8}{|c|}{$\phi$} \\
\hline & & & 1 & 5 & 16 & 25 & 50 & 75 & 84 & 95 \\
\hline \multicolumn{11}{|c|}{ Lacustrine plains } \\
\hline \multirow{3}{*}{ Moderately deep } & \multirow{3}{*}{1} & $0-20$ & -0.70 & 0.55 & $\overline{1.85}$ & 4.35 & 6.15 & 7.00 & 7.40 & 7.75 \\
\hline & & $20-60$ & -0.45 & 0.30 & 1.45 & 3.85 & 6.35 & 7.15 & 7.30 & 8.85 \\
\hline & & $60-90$ & -0.55 & 0.20 & 1.25 & 2.00 & 6.25 & 7.10 & 7.35 & 7.80 \\
\hline \multirow{7}{*}{ Deep } & \multirow{2}{*}{2} & $0-50$ & -0.20 & 0.85 & 3.35 & 5.30 & 6.85 & 7.55 & 7.70 & 8.10 \\
\hline & & $50-110$ & -0.20 & 0.85 & 3.45 & 5.40 & 6.90 & 7.60 & 7.70 & 8.10 \\
\hline & \multirow{2}{*}{3} & $0-45$ & -0.25 & 0.80 & 3.85 & 5.65 & 6.85 & 7.65 & 7.90 & 8.25 \\
\hline & & $45-110$ & -0.40 & 0.60 & 3.45 & 5.60 & 6.90 & 7.65 & 7.90 & 8.25 \\
\hline & \multirow{3}{*}{4} & $0-30$ & -0.60 & -0.10 & 0.55 & 1.05 & 6.20 & 7.15 & 7.45 & 7.95 \\
\hline & & $30-70$ & -0.70 & -0.30 & 0.35 & 0.80 & 5.90 & 6.95 & 7.30 & 7.85 \\
\hline & & $70-130$ & -0.80 & -0.50 & 0.15 & 0.60 & 5.20 & 6.65 & 6.95 & 7.60 \\
\hline \multirow{3}{*}{ Very deep } & \multirow{3}{*}{5} & $0-30$ & -0.85 & -0.70 & -0.40 & -0.20 & 0.35 & 4.10 & 5.55 & 6.85 \\
\hline & & $30-80$ & -0.80 & $\mid-0.60$ & -0.30 & -0.05 & 0.75 & 4.80 & 5.65 & 7.00 \\
\hline & & $80-160$ & -0.45 & 0.10 & 0.75 & 1.25 & 3.05 & 5.20 & 5.85 & 7.10 \\
\hline \multicolumn{11}{|c|}{ Windblown sand } \\
\hline \multirow{2}{*}{ Sand Sheet } & \multirow{2}{*}{6} & $0-50$ & $\begin{array}{c}-0.60 \\
\end{array}$ & \begin{tabular}{|c|}
-0.15 \\
\end{tabular} & 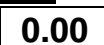 & 0.10 & 0.45 & 1.30 & 1.40 & 4.10 \\
\hline & & $50-100$ & -0.65 & -0.30 & 0.00 & 0.10 & 0.35 & 0.90 & 1.30 & 4.00 \\
\hline \multirow{4}{*}{ Barchan dunes } & \multirow[b]{2}{*}{$y$} & $0-20$ & -0.50 & -0.30 & -0.15 & -0.05 & 0.20 & 0.90 & 1.15 & 3.90 \\
\hline & & $20-100$ & -0.65 & -0.50 & -0.40 & -0.30 & -0.15 & 0.45 & 0.70 & 3.50 \\
\hline & \multirow{2}{*}{8} & $0-20$ & -0.75 & -0.55 & -0.35 & -0.30 & 0.05 & 0.60 & 0.80 & 2.15 \\
\hline & & $20-100$ & -0.75 & -0.50 & -0.30 & -0.30 & 0.15 & 0.75 & 1.00 & 3.00 \\
\hline \multirow{3}{*}{$\begin{array}{l}\text { Barchan dunes } \\
\text { partial cemented } \\
\text { with } \mathrm{CaCO}_{3}\end{array}$} & \multirow{3}{*}{9} & $0-40$ & -0.55 & -0.25 & -0.10 & 0.05 & 0.55 & 1.25 & 1.50 & 3.60 \\
\hline & & $40-80$ & -0.45 & -0.10 & -0.05 & 0.00 & 0.40 & 1.00 & 1.45 & 3.85 \\
\hline & & $80-120$ & -0.55 & \begin{tabular}{|l|}
-0.20 \\
\end{tabular} & -0.10 & 0.00 & 0.30 & 1.20 & 1.40 & 3.80 \\
\hline
\end{tabular}




\begin{tabular}{|c|c|c|c|c|c|c|c|c|c|c|}
\hline 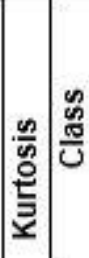 & & 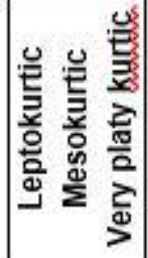 & $\begin{array}{ll}0 \\
0\end{array}$ & 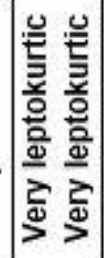 & 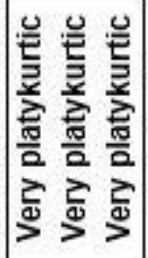 & 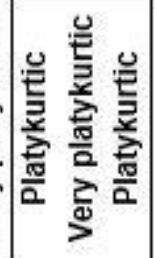 & 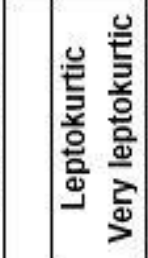 & 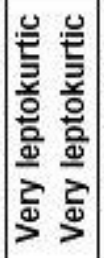 & 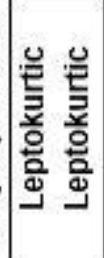 & 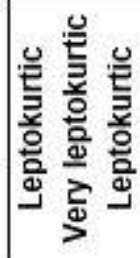 \\
\hline ב" & & 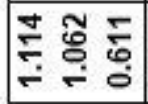 & స్ָ & స్ํำ & 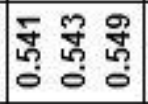 & 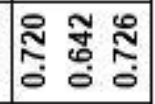 & 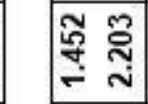 & 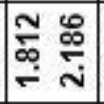 & స్్유. & 旁 \\
\hline 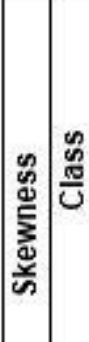 & & 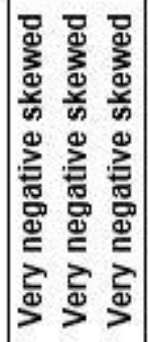 & 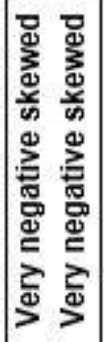 & 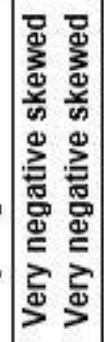 & 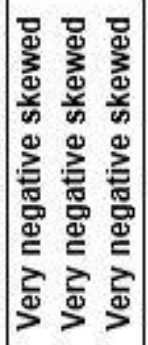 & 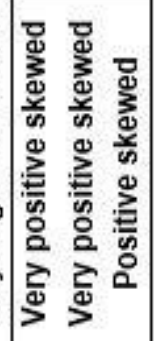 & 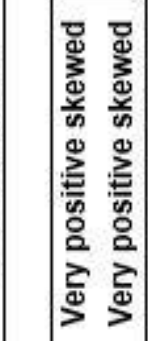 & 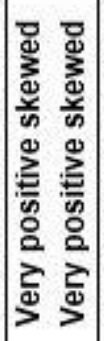 & 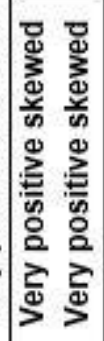 & 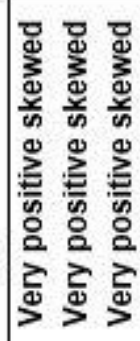 \\
\hline 㐫 & & 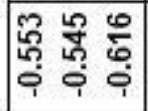 & 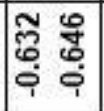 & 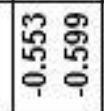 & $\mid \begin{array}{lll} & 9 & 0 \\
0 & 0 & 0 \\
0 & 0 & 0 \\
0 & 0 & 0\end{array}$ & مِ & 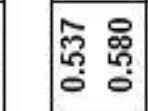 & 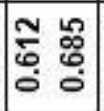 & 突 : & 总 \\
\hline 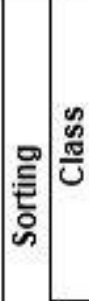 & 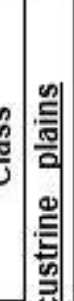 & 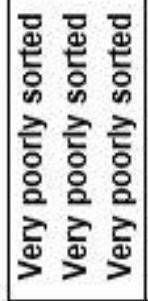 & 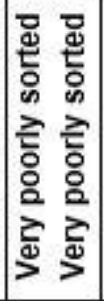 & 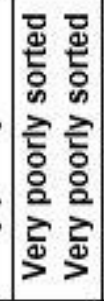 & 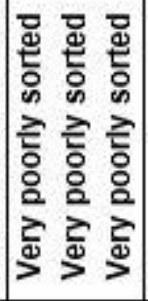 & 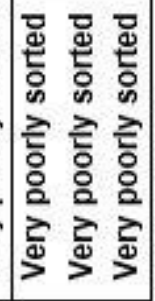 & 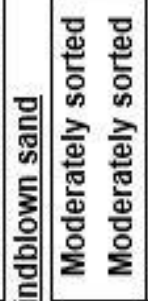 & 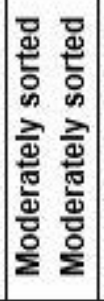 & 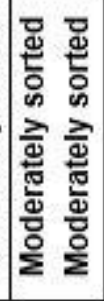 & 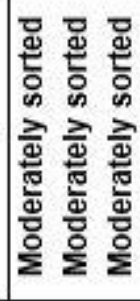 \\
\hline$\sigma$ & 8 & 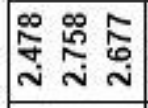 & 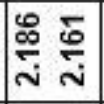 & 雲 & 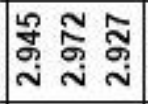 & 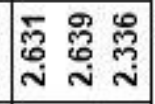 & 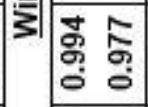 & 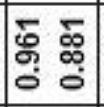 & 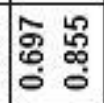 & ஜू. \\
\hline 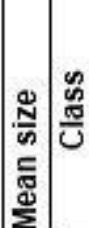 & : & 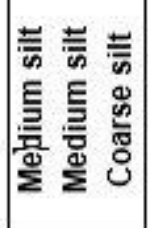 & 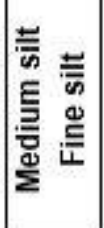 & 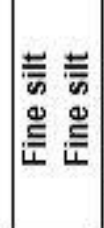 & 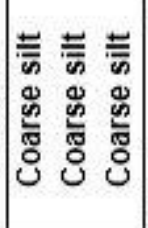 & 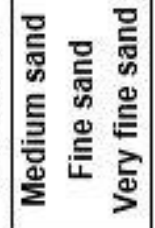 & 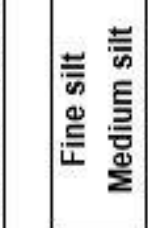 & 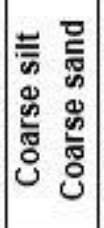 & 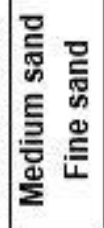 & 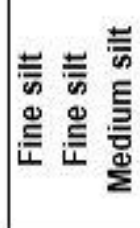 \\
\hline$\sum$ & $\frac{b}{8}$ & 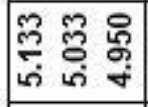 & 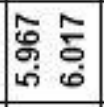 & 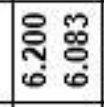 & 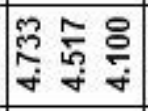 & 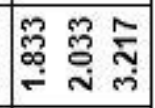 & 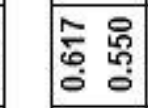 & 府 & 흄 & 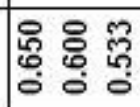 \\
\hline 홍형 & & 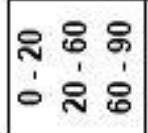 & 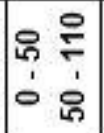 & 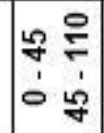 & 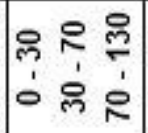 & $\begin{array}{llll}0 & 8 & 8 \\
m & 1 & 0 \\
1 & 1 & 1 \\
0 & 0 & 8\end{array}$ & $\begin{array}{|ll|}0 & 8 \\
0 & 0 \\
1 & 1 \\
0 & 5 \\
\end{array}$ & 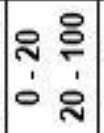 & 요문 & 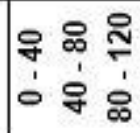 \\
\hline 을 옴 & & - & $\sim$ & $m$ & $\nabla$ & & 0 & $\sim$ & $\infty$ & $\sigma$ \\
\hline 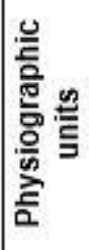 & & 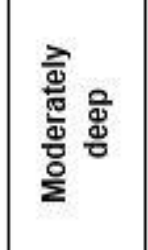 & & & & $\begin{array}{l}\text { 웡 } \\
\frac{0}{0} \\
\frac{1}{0} \\
\text { > }\end{array}$ & 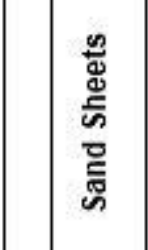 & 㟧 & & 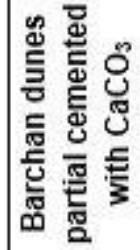 \\
\hline
\end{tabular}


Soils of lacustrine plains have values of Inclusive Graphic, Standard Deviation $\sigma 1$ between 2.141 and 2.972 o which reveal to very poorly sorted class. According to Inman (1952) sediments transported and deposited by water action or weathered sorted in situ are usually poorly sorted. On the other hand, Folk and Ward (1957) stated that, sorting is a sinusoidal function of mean size, so the values increase with transportation owing to decreases in the mean size of sediments.

Soils of windblown sand are characterized by moderate to moderately well sorted sediments throughout their depths. This indicates that, their sediments are transported and deposited under a combined action of both water and wind (Inman, 1952).

Sorting values $\sigma 1$ in Table (3), showed an improvement or better sorted, if they compared with Lacustrine plains, where their values varied between 0.697 and $0.994 \phi$ in windblown sand and from 2.141 to 2.977 o in lacustrine plains. The current results of the statistical size parameter showed the same conclusion of the study of Khatter and El-Toukhy (1986). They found that, this area have values of sorting between 1.00 and 0.50 o which attributed to aqueous and aeolian transportation. On the other hand, Labib and Khalil (1977) stated that, aeolian sandy depositions show better sorting relative to that finer one, while its values reveal to poorly sorted.

\subsection{Measure of skewness or asymmetry (Sk $\left.\mathbf{S}_{1}\right)$}

Skewness measures the degree of asymmetry of the frequency distributions and marks the position of the mean with respect to median. The symmetrical curve has $\mathrm{SK}_{\mathrm{I}}=\mathbf{0 . 0 0}$. Those with excess fine materials (a tail to the right) has positive skewness and these with excess coarse materials (a tail to the left) have negative skewness (Folk,1980 and Sahu, 1964).

Data in Table (3) pointed out that, soils of lacustrine plains have skewness (Sk1) values ranged between 0.446 and 0.646 o in all representative profiles except soils of profile 4, which are corresponding strongly coarse skewed that is the mean is towards the coarse side of the median. While in soils of profile 5 have skewness values of strongly fine skewed in surface and subsurface layers and fine skewed in the deepest one, which indicate that, the studied samples have tails of fine grains.

Soils of windblown sand have values of skewness (Sk1) differ from 0.386 to 0.685 中 which are related with strongly fine skewed class, which indicate that these soil samples have tails of fine grains.

\subsection{Measure of Kurtosis or Peakedness $\left(\mathrm{K}_{\mathrm{G}}\right)$ :}

Kurtosis measures the ratio between the sorting in the "tails" of the curve and the sorting in the central portion. If the central portion is better sorted than the tail, the curve is said to be excessively peaked or leptokurtic. If the tails are better sorted than central portion, the curve is deficiently or flat -peaked and platykurtic (Folk, 1980 and Shau, 1964).

Soils of Lacustrine plains appear kurtosis values (Table, 3) as platykurtic and very platykurtic in soils of profiles 4,5 and deepest layer of profile 1. This indicates that, water is the main factor responsible for soil formation. Soils of profiles $2 \& 3$ and surface layer of profile 1 have leptokurtic and very leptokurtic classes, which indicate that, the involvement of wind and water actions in the formation of soils. Subsurface layer of profile 1 has mesokurtic class which reveals to the previous conclusion. 
A. M. A. Zayed, et al.,

Soils of windblown sand have leptokurtic and very leptokurtic classes in all representative profiles which emphasize union of its mechanisms and environments of deposition and are formed under both wind and water actions.

\section{Depositional environment:}

According to Sahu (1964) every environment of deposition can be assumed to have its characteristic energy conditions and energy fluctuations through space and time. The preservation of these fluctuations is subject to the availability of sufficient amounts of source material of all sizes. If so, then size distribution would indicate the environment of deposition.

Data in Table (4) showed that, all samples of the studied soils have values of $Y$ aeol: beoch greater than -2.7411 which reveal to the environment isn't aeolian process. Values of $\mathrm{Y}$ beach: sh.mar. were greater than 65.5650 don't indicate littoral (beach) deposition environment, except $0-20 \mathrm{~cm}$ layer of profile 8 that has $Y$ beach: sh.mar. value less than 65.3650 indicating beach deposition environment. Data of $Y$ sh: mar.: fluv., of rest layer samples, are less than-7.4190 indicating a fluvial (deltic) environment.

Generally, values of $Y$ aeol: beoch and $Y$ beach: sh.mar. of windblown sand unit are less than Lacustrine plains and opposite trend is recorded in $Y$ sh: mar.: fluv. and $Y$ fluv.: turb. which may be reveal to presence of another agent of deposition environment of soils for windblown sand.

Table (4): The depositional environments of the studied soils according to Sahu (1964).

\begin{tabular}{|c|c|c|c|c|c|c|}
\hline Landform & $\begin{array}{l}\text { Profile } \\
\text { No. }\end{array}$ & $\begin{array}{l}\text { Depth } \\
\text { Cm }\end{array}$ & $\begin{array}{c}Y \\
\text { aeol: } \\
\text { beoch }\end{array}$ & $\begin{array}{c}Y \\
\text { beach: } \\
\text { sh.mar. }\end{array}$ & $\begin{array}{c}Y \\
\text { sh.mar.: } \\
\text { fluv. }\end{array}$ & $\begin{array}{l}\text { Y } \\
\text { fluv.: } \\
\text { turb. }\end{array}$ \\
\hline \multicolumn{7}{|c|}{ Lacustrine plains } \\
\hline $\begin{array}{l}\text { Moderately } \\
\text { deep }\end{array}$ & 1 & $\begin{array}{c}0-20 \\
20-60 \\
60-90\end{array}$ & $\begin{array}{c}9.0316 \\
14.6308 \\
12.0319\end{array}$ & $\begin{array}{l}494.5728 \\
588.3694 \\
548.3591\end{array}$ & $\begin{array}{l}-49.5894 \\
-62.4798 \\
-58.3031\end{array}$ & $\begin{array}{r}3.4019 \\
2.5158 \\
-0.2283\end{array}$ \\
\hline \multirow[t]{3}{*}{ Deep } & 2 & $\begin{array}{c}0-50 \\
50-110\end{array}$ & $\begin{array}{l}1.8188 \\
1.3607\end{array}$ & $\begin{array}{l}420.3810 \\
414.3235\end{array}$ & $\begin{array}{l}-37.0031 \\
-35.9665\end{array}$ & $\begin{array}{l}5.1127 \\
5.2567\end{array}$ \\
\hline & 3 & $\begin{array}{c}0-45 \\
45-110\end{array}$ & $\begin{array}{l}0.7469 \\
3.3958\end{array}$ & $\begin{array}{l}416.5742 \\
451.7496\end{array}$ & $\begin{array}{l}-35.6205 \\
-40.4660\end{array}$ & $\begin{array}{l}6.9838 \\
6.3729\end{array}$ \\
\hline & 4 & $\begin{array}{c}0-30 \\
30-70 \\
70-130\end{array}$ & $\begin{array}{l}18.1380 \\
19.4364 \\
19.7219\end{array}$ & $\begin{array}{l}642.9897 \\
651.1543 \\
629.3066\end{array}$ & $\begin{array}{l}-71.6445 \\
-73.3458 \\
-71.6874\end{array}$ & $\begin{array}{l}-1.2659 \\
-1.1924 \\
-0.5959\end{array}$ \\
\hline Very deep & 5 & $\begin{array}{c}0-30 \\
30-80 \\
80-160\end{array}$ & $\begin{array}{l}19.8032 \\
19.1811 \\
10.7091\end{array}$ & $\begin{array}{l}510.3209 \\
513.0322 \\
424.5484 \\
\end{array}$ & $\begin{array}{l}-63.6995 \\
-63.5606 \\
-47.4604\end{array}$ & $\begin{array}{l}7.2881 \\
6.4078 \\
4.8255 \\
\end{array}$ \\
\hline \multicolumn{7}{|c|}{ Windblown sand } \\
\hline Sand Sheets & 6 & $\begin{array}{c}0-50 \\
50-100\end{array}$ & $\begin{array}{l}4.8594 \\
7.2220\end{array}$ & $\begin{array}{l}111.1577 \\
122.5259\end{array}$ & $\begin{array}{l}-11.0383 \\
-10.9269\end{array}$ & $\begin{array}{l}11.3470 \\
15.5737\end{array}$ \\
\hline \multirow[t]{2}{*}{ Barchan dunes } & 7 & $\begin{array}{c}0-20 \\
20-100\end{array}$ & $\begin{array}{l}6.3646 \\
8.0775 \\
\end{array}$ & $\begin{array}{l}111.5956 \\
104.6446 \\
\end{array}$ & $\begin{array}{l}-10.8884 \\
-10.0338 \\
\end{array}$ & $\begin{array}{l}13.6243 \\
15.9051\end{array}$ \\
\hline & 8 & $\begin{array}{c}0-20 \\
20-100\end{array}$ & $\begin{array}{l}4.1366 \\
4.9780 \\
\end{array}$ & $\begin{array}{l}65.0299 \\
86.2598 \\
\end{array}$ & $\begin{array}{l}-6.2479 \\
-8.5526 \\
\end{array}$ & $\begin{array}{c}9.3266 \\
10.2916 \\
\end{array}$ \\
\hline $\begin{array}{l}\text { Barchan dunes } \\
\text { partial cemented }\end{array}$ & 9 & $\begin{array}{c}0-40 \\
40-80\end{array}$ & $\begin{array}{l}4.5520 \\
5.2162\end{array}$ & $\begin{array}{l}105.0315 \\
112.0014\end{array}$ & $\begin{array}{l}-10.1106 \\
-10.8587\end{array}$ & $\begin{array}{c}9.6370 \\
12.4795\end{array}$ \\
\hline
\end{tabular}




\begin{tabular}{l|l|l|l|l|l|l} 
with $\mathrm{CaCO}_{3}$ & & $80-120$ & 4.6495 & 107.8864 & -11.1905 & 11.3228 \\
\hline
\end{tabular}

\section{Mechanism of transportation:}

According to Passega (1957 and 1964), the C-M pattern (Fig, 3) is used as a tool for indicating the hydrodynamic conditions of sedimentation and divided the C-M diagram into 6 classes depending on the relationship between one percentile and median in microns.

Data in Fig (3) and Table (5) showed that, soils of lacustrine plains have $T$ segment (pelagic suspension) except soils of profile 5, which appear mechanism of transportation differ from layer to another. Whereas in the surface layer is rolling ( $\mathrm{N}-\mathrm{O}$ segment), rolling and suspension (O-P segment) in subsurface layer, while the deepest layer appears graded suspension (Q-R segment) which reflect the fineness of particles with depth. Soils of windblown sand fall in NO segment, which reveal to mechanism of transportation is by rolling only in all representative profile layers. This is except for the surface layer of profile 9 which belong to O-P segment, and reveal to rolling and suspension mechanism of transportation and nearer to $\mathrm{N}-\mathrm{O}$ segment or on its left edge.

These data are classified in C-M pattern diagram into two clusters, one in the right of diagram which represents the windblown soils. Another fall in the left of C-M diagram which represents the lacustrine plains, that emphasize the differences of means and mechanism of transportation and deposition.

Furthermore, the available data of the statistical size parameters reveal that, the studied soil profiles are of non-uniform parent materials. However, the stratified conditions observed in these profiles is mostly attributed to different depositional materials and/or depositional regime.

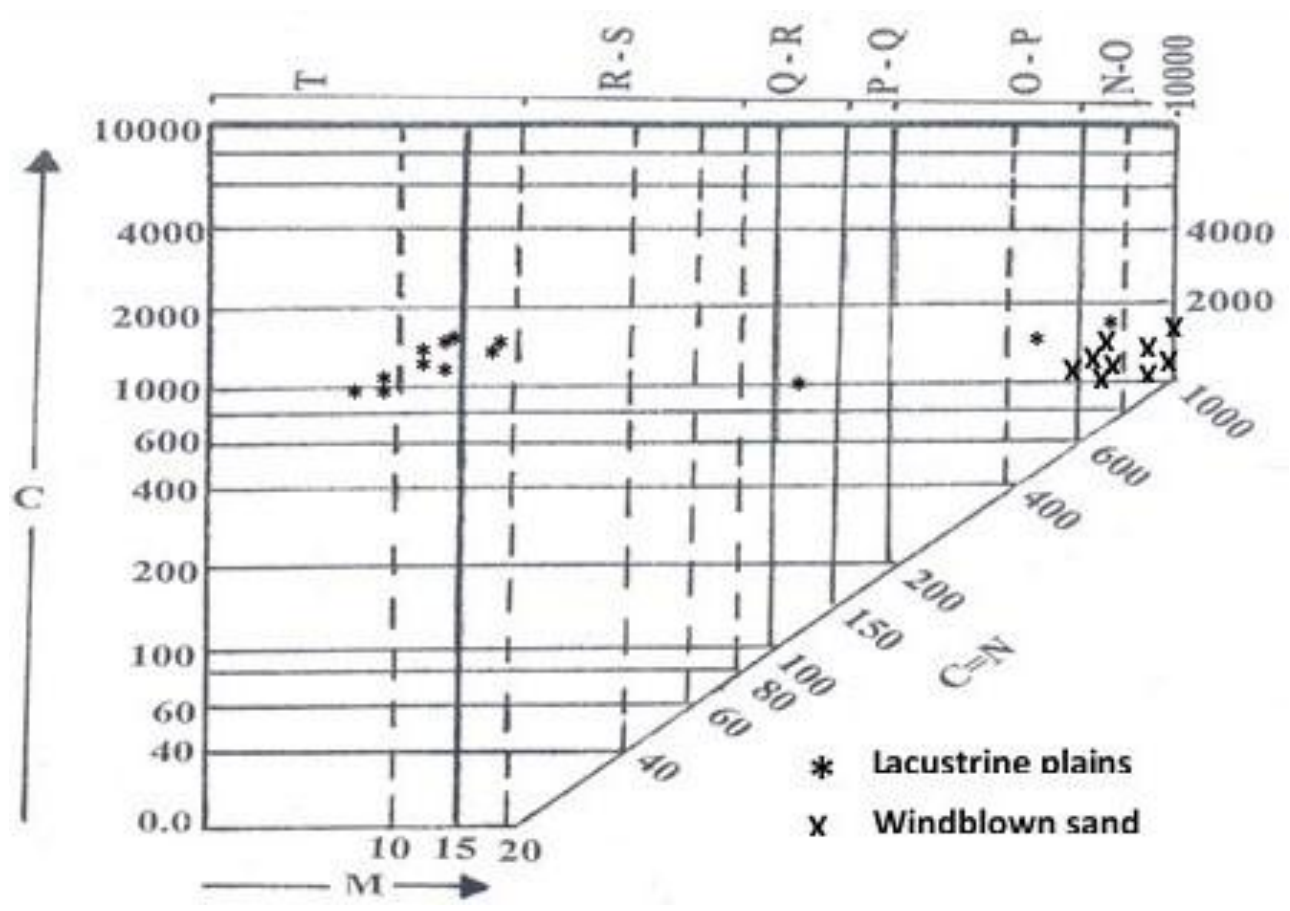

Fig (3): C-M Pattern diagram for hydrodynamic conditions of the studied soils. 
A. M. A. Zayed, et al.,

Table (5): One percentile, median size and hydrodynamic conditions of the studied area.

\begin{tabular}{|c|c|c|c|c|c|c|}
\hline \multirow{2}{*}{$\begin{array}{l}\text { Physiographic } \\
\text { units }\end{array}$} & \multirow{2}{*}{$\begin{array}{l}\text { Profile } \\
\text { No. }\end{array}$} & \multirow{2}{*}{$\begin{array}{l}\text { Depth } \\
\text { (cm) }\end{array}$} & \multirow{2}{*}{$\begin{array}{c}\text { One } \\
\text { percentile } \\
\text { (micron) }\end{array}$} & \multirow{2}{*}{$\begin{array}{l}\text { Median } \\
\text { diameter } \\
\text { (micron) }\end{array}$} & \multicolumn{2}{|c|}{ Mechanism of transportation } \\
\hline & & & & & Segment & Indication \\
\hline \multicolumn{7}{|c|}{ Lacustrine plains } \\
\hline \multirow{3}{*}{$\begin{array}{l}\text { Moderately } \\
\text { deep }\end{array}$} & \multirow{3}{*}{1} & $0-20$ & 1626 & 14.43 & $T$ & Pelagic suspension \\
\hline & & $20-60$ & 1366 & 14.87 & $\mathbf{T}$ & Pelagic suspension \\
\hline & & $60-90$ & 1464 & 13.65 & $\mathbf{T}$ & Pelagic suspension \\
\hline \multirow{7}{*}{ Deep } & \multirow{2}{*}{2} & $0-50$ & 1152 & 8.97 & $\mathbf{T}$ & Pelagic suspension \\
\hline & & $50-110$ & 1152 & 8.58 & $\mathbf{T}$ & Pelagic suspension \\
\hline & \multirow{2}{*}{3} & $0-45$ & 1190 & 8.97 & $\mathbf{T}$ & Pelagic suspension \\
\hline & & 45- 110 & 1322 & 8.58 & $\mathbf{T}$ & Pelagic suspension \\
\hline & \multirow{3}{*}{4} & $0-30$ & 1518 & 14.04 & $\mathbf{T}$ & Pelagic suspension \\
\hline & & $30-70$ & 1626 & 17.14 & $\mathbf{T}$ & Pelagic suspension \\
\hline & & $70-130$ & 1744 & 18.68 & $\mathbf{T}$ & Pelagic suspension \\
\hline \multirow{3}{*}{ Very deep } & \multirow{3}{*}{5} & $0-30$ & 1808 & 788 & $\mathrm{~N}-\mathrm{O}$ & Rolling \\
\hline & & $30-80$ & 1744 & 590 & O-P & Rolling \& suspension \\
\hline & & $80-160$ & 1366 & 121 & Q-R & Graded suspension \\
\hline \multicolumn{7}{|c|}{ Windblown sand } \\
\hline \multirow{2}{*}{ Sand Sheets } & \multirow{2}{*}{6} & $0-50$ & 1518 & 736 & N-O & Rolling \\
\hline & & $50-100$ & 1572 & 788 & $\mathrm{~N}-\mathrm{O}$ & Rolling \\
\hline \multirow{4}{*}{ Barchan dunes } & \multirow[b]{2}{*}{7} & $0-20$ & 1410 & 808 & N- $O$ & Rolling \\
\hline & & $20-100$ & 1572 & 1114 & $\mathrm{~N}-\mathrm{O}$ & Rolling \\
\hline & \multirow[b]{2}{*}{8} & $0-20$ & 1680 & 968 & $\mathrm{~N}-\mathrm{O}$ & Rolling \\
\hline & & $20-100$ & 1680 & 904 & $\mathrm{~N}-\mathrm{O}$ & Rolling \\
\hline \multirow{3}{*}{$\begin{array}{l}\text { Barchan dunes } \\
\text { partial cemented } \\
\text { with } \mathrm{CaCO}_{3}\end{array}$} & \multirow{3}{*}{9} & $0-40$ & 1464 & 566 & O-P & Rolling \& suspension \\
\hline & & $40-80$ & 1366 & 762 & $\mathrm{~N}-\mathrm{O}$ & Rolling \\
\hline & & $80-120$ & 1464 & 814 & N- $O$ & Rolling \\
\hline
\end{tabular}

\section{REFERENCES}

Burt, R. E. (2004). " Soil Survey Laboratory Methods Manual", Soil Survey Investigation Report No. 42 Version 4.0.

Doeglas, D. G. (1946). Interpretation of results of mechanical analysis. J. Sedimentary Petrology, 16: 19-40.

Folk, R. L. (1980). Petrology of Sedimentary Rocks" Geology Hemphill Publishing Company, Austin, Taxas 78703.

Folk, R. L. and W. C. Ward (1957). Barazos River Bar. A study in the significance of grain size parameters. J. of Sedimentary Petrology, 27 (1): 326.
Griffiths, J. C. (1967). Scientific method in analysis of sediments. Mc. Graw-Hill Book Comp., New York, St. Louis, USA.

Inman, D. L. (1952). Measures for describing the size distribution of sediments. J. Sedimentary Petrology, 22: 125-145.

Khatter, E. A. and A. A. EL- Toukhy (1986). Nature and characteristics of some new extension scopes adjacent to the cultivated soils at different desertic region of Egypt, Bull. Fac. of Agric., Univ. of Cairo, 37 (1): 411-424.

Labib, F. and J. B. Khalil (1977). Pedological study of some sediments 
in the Western Desert. Egypt, J. Soil Sci. 17 (2): 203-221.

Passega, R. (1957). Texture as characteristic of clastic deposition. Am. J. Assoc. Petroleum Geologist, 41 (9): 1952-1984.

Passega, R. (1964). Grain size representation by $C-M$ pattern as geological tool. J. Sed. Petrol., 34 (4): 830-847.

Piper, C. S. (1950). "Soil and Plant Analyses", Inter science Publisher Inc. New York, USA.
Sahu, B. K. (1964). Dispositional mechanisms from the size analysis of clastic sediments. J. of Sedimentary Petrology, 34 (1): 73 - 83.

Visher, S. G. (1969). Grain size distribution and depositional processes, J. of Sedimentary Petrology, 39 (3): 1074-1106.

Zayed, A. M. R., H. M. A. El-Tabey and A. A. Al-Toukhy (2020). Study of some soils South El-Amiria, Alexandria Governorate, Egypt, using two soil classification systems, Egypt. J. Agric. Res., 98 (3): 548 - 558. 
A. M. A. Zayed, et al.,

$$
\text { الخصائص الترسيبية لأراضي جنوب العامرية - محافظه الأسكندرية - مصر }
$$

عادل محمد عبد الرحمن زايل، هانئ محمد أحمد التابعي، عبد المنعم عبد المجيد الطوخي معهذ بحوث الأراضي والمياه والبيئة - مركز البحوث الزراعية - الجيزة - مصر

الملخص العربي

تهاف هذه الاراسة إلى التعرف على الخصائص الترسيبية لأراضي جنوب العامرية وذلك من خلال دراسة التحليل الإحصائي لحجوم حبييات التربة ، وييئات الترسيب وميكانيكيات النقل و تتميز منطقة الدراسة بوجود وحلتان فيزيوجرافيتان

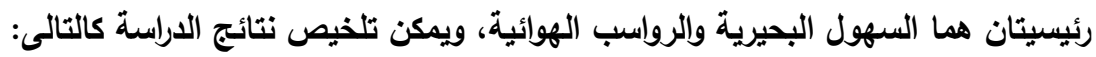

$$
\text { 1- أراضي السهول البحيرية: }
$$

وكانت نتائج التحليل الإحصائي لحجوم حبيبات التربة كما يلي:

• دلت قيم الوسيط Median (Md) على أن القطر المتوسط للحبيبات هو السلت الناعم كمكون رئيسي، بينما كان

$$
\text { المتوسط البياني (Mz) على وجه العموم بين السلت الناعم والمتوسط والخشن. }
$$

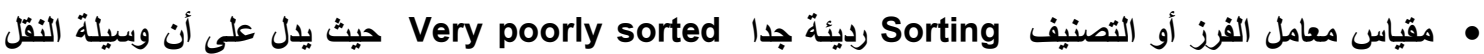

$$
\text { والترسيب للأراضي كانت المياه. }
$$

• وقد أوضح مقياس التناسق أو الحيود Skewness أن الترسيبات ذات قيم Strongly coarse skewed وأن

$$
\text { المتوسط يميل إلى الحبيبات الخشنة. }
$$

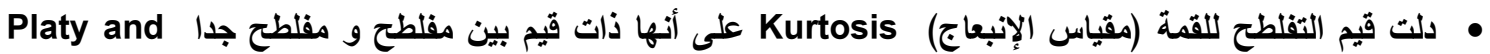
very platykurtic بالإضافة إلى Lepto and very leptokurtic مدبب و مدبب جدا.

• • نتائج بيئة الترسيب لهذه الوحدة كانت دلتاوية Fluvial or deltaic بواسطة المياه. • ميكانيكيه النقل طبقا للنموذج الهنسي C-M كانت بواسطة المعلق المتجانس Pelagic suspension على وجه

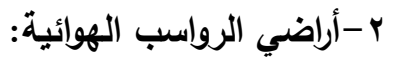

وكانت نتائج التحليل الإحصائي لحجوم حبييات التربة كما يلي: • الرمل الخشن لكل من مقياسي الوسيط (Md) والمتوسط البياني (Mz).

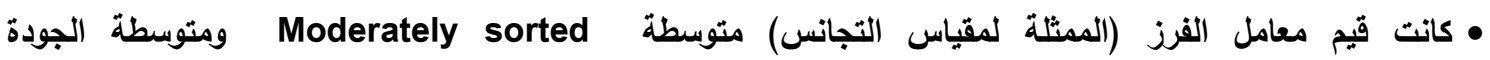
Moderately well sorted • مقياس التناست لتوزيع هذه الترسييات كان Strongly coarse skewed وأن المتوسط يميل إلى الحبيبات الناعمة. • دل مقياس تفلطح القمه على أنها ذات قيم بين مفلطح و مفلطح جدا Platy and very platykurtic.

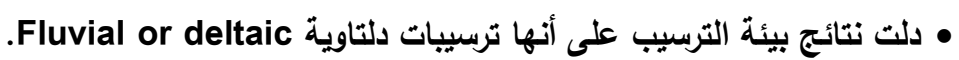
• ميكانيكيه النقل بتأثير الاحرجة.

$$
\begin{aligned}
& \text { معهل بحوث الأراضى والمياه - قسم حصر وتصنيف الأراضى } \\
& \text { أ.د / حسنى حسين حسونـــــة } \\
& \text { أ.د/ محمد سمير عراقى عميره كلية الزراعة - جامعة المنوفية الاضية }
\end{aligned}
$$

